\title{
Acute Hemorrhagic Retinopathy following Intravitreal Melphalan Injection for Retinoblastoma: A Report of Two Cases and Technical Modifications to Enhance the Prevention of Retinal Toxicity
}

\author{
Hassan A. Aziz ${ }^{a}$ Jonathan W. Kim ${ }^{a} \quad$ Francis L. Munier ${ }^{b}$ Jesse L. Berry ${ }^{a}$ \\ aThe Vision Center at Children's Hospital Los Angeles, USC Roski Eye Institute, Keck School of Medicine, \\ University of Southern California, Los Angeles, Calif., USA; ${ }^{b}$ Department of Ophthalmology, Jules Gonin \\ Eye Hospital, Lausanne, Switzerland
}

\section{Key Words}

Chorioretinal toxicity Intravitreal melphalan .

Retinoblastoma - Vascular occlusion · Vitreous seeding

\begin{abstract}
Aims: To report the occurrence of acute hemorrhagic retinopathy following intravitreal melphalan injection for retinoblastoma. Methods: This is a retrospective case series of 2 patients with retinoblastoma treated with intravitreal melphalan for vitreous seeding who developed acute hemorrhagic retinopathy. Results: Patient 1 is a 6-month-old female with bilateral retinoblastoma (Group D right eye and Group B left eye) treated with 4 cycles of systemic chemotherapy and 2 intravitreal melphalan injections in each eye. Patient 2 is a 10-month-old male with unilateral Group D retinoblastoma treated with 6 cycles of systemic chemotherapy and 2 injections of intravitreal melphalan. At the 1-week follow-up after the second injection, both patients had an acute hemorrhagic retinopathy that resulted in chorioretinal toxicity with a sharp demarcation line between the normal and abnormal retina. At the last follow-up ( 22 and 12 months, respectively), there was total tumor control and resolution of vitreous seeding in both patients. Conclusions: Although in-
\end{abstract}

(C) 2016 S. Karger AG, Basel

E-Mail karger@karger.com www.karger.com/oop travitreal melphalan injection is effective for vitreous seeding in eyes with retinoblastoma, acute hemorrhagic retinopathy and diffuse chorioretinal atrophy is a possible complication of this treatment modality. Given the clinical findings observed in these patients, the development of this retinal toxicity most likely results from a retrohyaloid overdose. Consequently we suggest preventive measures aimed at limiting the risk of retrohyaloid injection.

(c) 2016 S. Karger AG, Basel

\section{Introduction}

In 1962, Ericson and Rosengren [1] first described the use of intravitreal chemotherapy in retinoblastoma, but reports of extraocular tumor spread limited the use of this treatment modality. While there has been tremendous success in the last decade in globe salvaging therapy for advanced retinoblastoma, persistently active or recurrent vitreous seeding remains the most common reason for therapeutic failure [2]. After modification of the drug delivery technique in 2012, intravitreal melphalan injection has emerged as an effective therapy for vitreous seeding in retinoblastoma, with an overall acceptable safety profile [3-5]. 

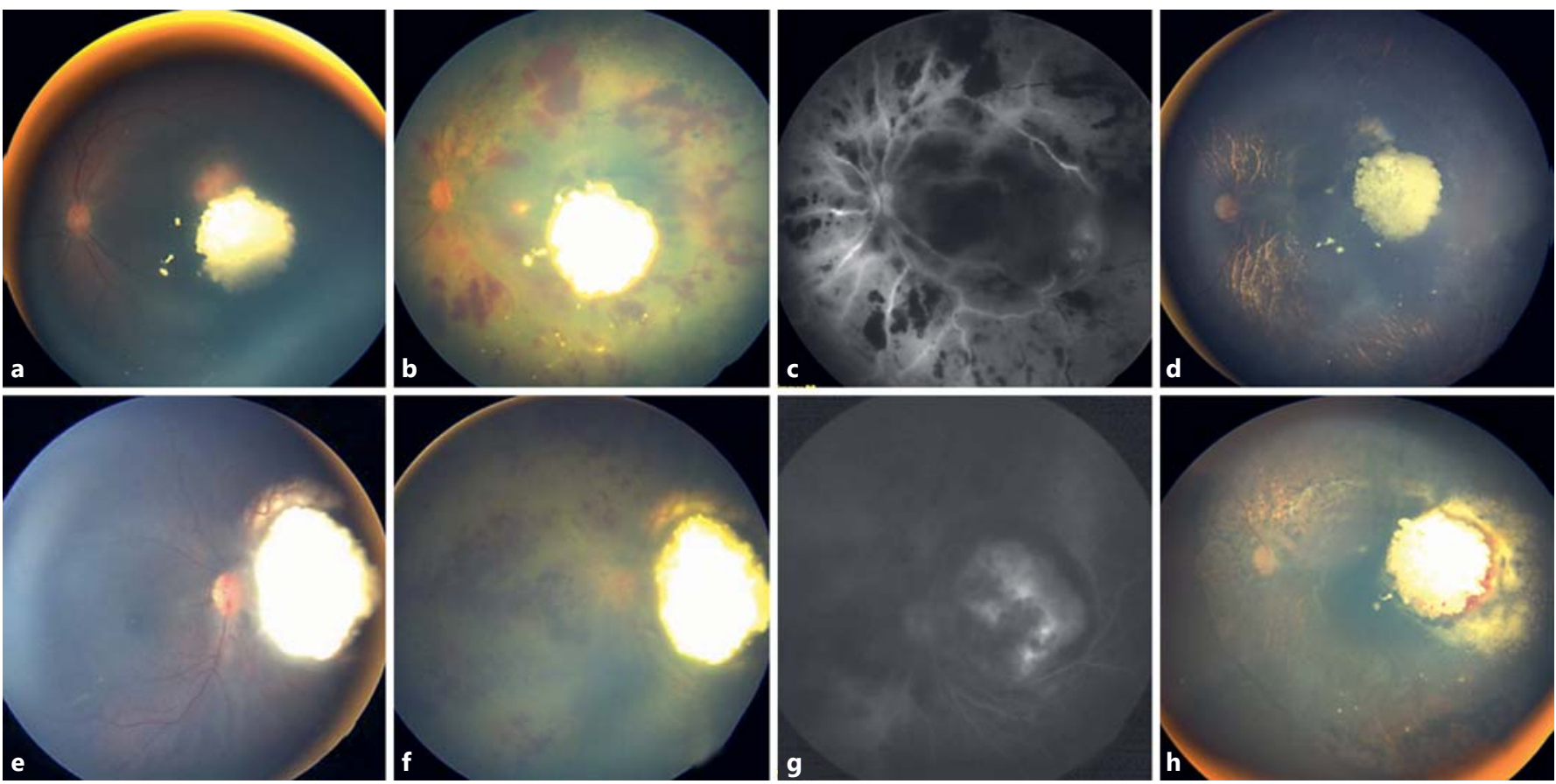

Fig. 1. a, e Fundus photographs of the left eye of patient 1 (a) and the right eye of patient 2 (e) revealing a partially treated retinoblastoma with associated vitreous seeding located in the macula and nasal to the optic disc, respectively. $\mathbf{b}$, $\mathbf{f}$ Fundus photographs of patient 1 (b) and patient 2 (f) revealing diffuse retinal edema with associated intra- and preretinal hemorrhages. c, $\mathbf{g}$ Mid-phase fluorescein angiography of the left eye of patient 1 (c) and the right eye

The most well-established and expected side effect of intravitreal melphalan injection is peripheral, localized chorioretinal toxicity. Munier [4] described varying degrees of salt-and-pepper retinopathy, which occurs at the site of injection. The clinical grading system ranges from localized salt-and-pepper retinopathy at the anterior injection site (grade I) to diffuse chorioretinal toxicity with optic atrophy (grade V) [4].

Other side effects have been described. For example, Francis et al. [3] demonstrated that weekly injections of melphalan resulted in a dose-dependent decrease in the electroretinogram (ERG) response in both humans and rabbits; thus, they cautioned against doses higher than 30 $\mu \mathrm{g}$. Ghassemi and Amoli [6] described ischemic necrosis in the retina and choroid, severe gliosis and phthisis bulbi, and secondary neovascularization in a histopathologic analysis of enucleated eyes after receiving $50 \mu \mathrm{g}$ of intravitreal melphalan. Severe toxicity after intravitreal melphalan injection appears to be rare. Smith et al. [7] performed a meta-analysis of 1,287 intravitreal melphalan injections in 295 patients, and found only 17 patients with

of patient $2(\mathbf{g})$ revealing areas of blockage corresponding to the areas of retinal hemorrhage and intraocular tumor, with vascular sheathing. d, h Fundus photographs at the follow-up of patient 1 (d) and patient 2 (h) revealing diffuse chorioretinal atrophy in the posterior pole with a demarcation between the normal and the atrophic retina.

significant ocular side effects including iris atrophy, chorioretinal atrophy, vitreous hemorrhage, and retinal detachment.

Herein, we describe 2 cases of acute hemorrhagic retinopathy leading to diffuse chorioretinal atrophy following intravitreal melphalan injection. While the mechanism for this toxicity has not been fully elucidated, we propose that the vitreoretinal interface may play a role in the development of this toxicity.

\section{Case Series}

This is an interventional case series describing 2 patients who developed acute toxicity after intravitreal injection of melphalan as treatment for vitreous seeding. The Institutional Review Board at the Children's Hospital Los Angeles (CHLA) approved this review.

\section{Patient 1}

A 2-month-old female was diagnosed at the CHLA with bilateral retinoblastoma, Group D in the right eye and Group B in the left. Both eyes displayed a primarily endophytic growth pattern. 
The patient subsequently received 4 cycles of systemic chemotherapy and consolidation with focal laser therapy. After this treatment, at 6 months of age, she was noted to have persistently active vitreous seeding in the right eye and seeding from a recurrence in the left eye. It was decided to proceed with intravitreal melphalan injection in both eyes following the standard technique previously described by Munier [4]: a paracentesis is performed, withdrawing $0.1 \mathrm{ml}$ of aqueous humor; an injection is done $3.25-3.5 \mathrm{~mm}$ from the limbus with a 32 -gauge, 12 - $\mathrm{mm}$ ( 0.5 -inch) needle in a quadrant of the eye free of tumor; the needle is visualized behind the lens in the mid-anterior vitreous, and cryotherapy is applied as the needle is withdrawn from the eye; the eye is then shaken to distribute the chemotherapy, and the surface bathed in sterile water. The patient tolerated the first cycle of bilateral intravitreal melphalan injections with $30 \mu \mathrm{g}$ for the right eye and $25 \mu \mathrm{g}$ for the left eye without complication. The concentration of melphalan used was $25 \mu \mathrm{g} / 0.1$ $\mathrm{ml}$. A second cycle of bilateral injections was given 1 week later at the same dose (fig. 1a). A lower dose was chosen for the left eye, as the seeding burden was lower in this eye. The intraocular pressure was normal before and after the injection.

\section{Patient 2}

A 6-month-old male was diagnosed at the CHLA with unilateral Group D retinoblastoma in the right eye. The patient subsequently received 6 cycles of systemic chemotherapy and consolidation with focal laser therapy. Once the systemic chemotherapy had been completed, there remained persistently active vitreous seeding. Therefore it was decided to treat the eye with an intravitreal injection of melphalan. He was 10 months of age at the time of the injection. The patient underwent 1 cycle of intravitreal melphalan injection at a dose of $25 \mu \mathrm{g}(25 \mu \mathrm{g} / 0.1 \mathrm{ml})$ per the injection protocol described above. One week later there were no signs of complications or toxicity; therefore a second injection was given at the same dose (fig. 1e). The intraocular pressure was noted to be normal before and after the injection, and no complications or irregularities were noted at the time of the procedure.

\section{Patients 1 and 2}

The patients presented 1 week later for the third planned intravitreal injection, and both of them were noted to have diffuse hemorrhagic retinopathy with retinal edema, as well as intra- and preretinal hemorrhages (fig. $1 \mathrm{~b}, \mathrm{f}$ ). The toxicity was seen in only the left eye of patient 1 , despite the fact that she had received bilateral injections. Mid-phase fluorescein angiography of the involved eyes showed blockage secondary to the retinal tumor and the retinal hemorrhages, vascular sheathing, and nonperfusion with patchy filling of the choroid (fig. 1c, g, 2). The third injection was deferred given the presence of toxicity. At the 1-month follow-up, diffuse chorioretinal atrophy with a sharp demarcation between the atrophied and the normal retina was seen (fig. 1d, h). The ERG revealed a flat signal in the affected eyes of both patients, and, clinically, the eyes appeared to have poor vision. A post-injection B-scan revealed posterior vitreous detachment in both eyes (fig. 3). Unfortunately a B-scan had not been done immediately prior to initiating the injections; however, a B-scan performed at diagnosis for patient 2 did show an attached hyaloid at that time (fig. 3). Optical coherence tomography (OCT) conducted before the injection in patient 2 showed a normal foveal contour without direct involvement by the tumor or seeding. A subsequent OCT performed 2 months after the toxicity had been noted showed diffuse inner and

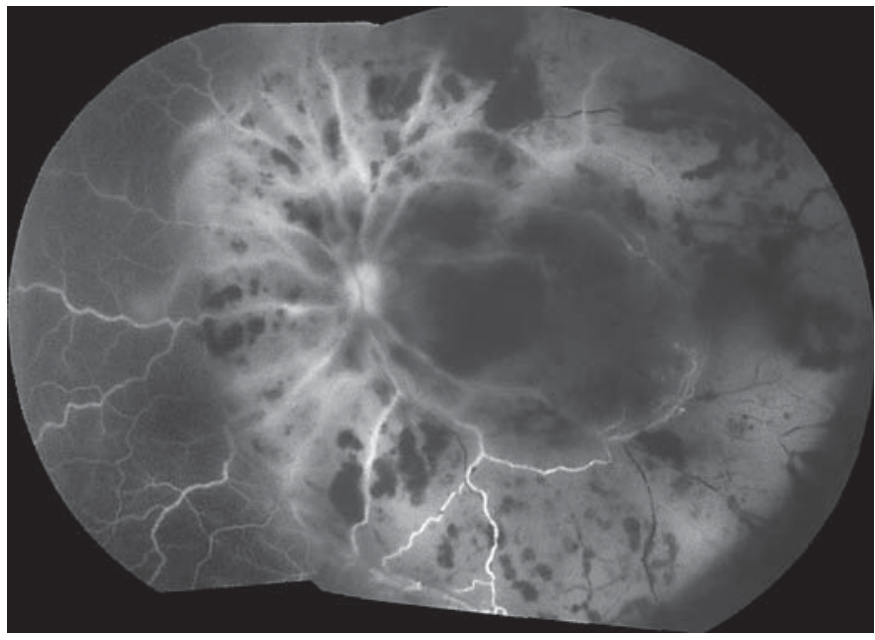

Fig. 2. Mid-phase fluorescein angiography montage of the left eye of patient 1 showing areas of hypofluorescence corresponding to retinal hemorrhages with widespread vascular sheathing, peripheral vascular occlusion, and capillary nonperfusion. This suggests that a vasoocclusive event, possibly secondary to an increased drug concentration at the neurosensory retina, may have played a role in toxicity.

outer retinal atrophy, loss of the foveal depression, as well as atrophy of the underlying choroid (fig. 4).

Neither patient required any further systemic or local treatment after the second injection for either the seeding or retinal tumors. At the 22-month and 12-month follow-up for patient 1 and patient 2 , respectively, the retinal tumors and vitreous seeds were regressed with no evidence of active disease.

\section{Discussion}

Persistent or recurrent vitreous seeding is a major cause of treatment failure in retinoblastoma [2]. The blood-retinal barrier restricts the bioavailability of antineoplastic agents within the vitreous cavity, limiting the effectiveness of either systemic or intra-arterial chemotherapy against vitreous seeding. Intravitreal melphalan injections directly bypass this barrier and achieve tumoricidal concentrations in the vitreous [4]. While highly effective, intravitreal melphalan injection may in rare cases be associated with serious ocular side effects $[6,7]$. The case series herein described presents the first examples of acute hemorrhagic retinal toxicity noted within 1 week of intravitreal melphalan injection, without any recognized deviation from the standard technique.

Recently, Suzuki et al. [8] described their 21-year experience with intravitreal melphalan injections for vitre- 

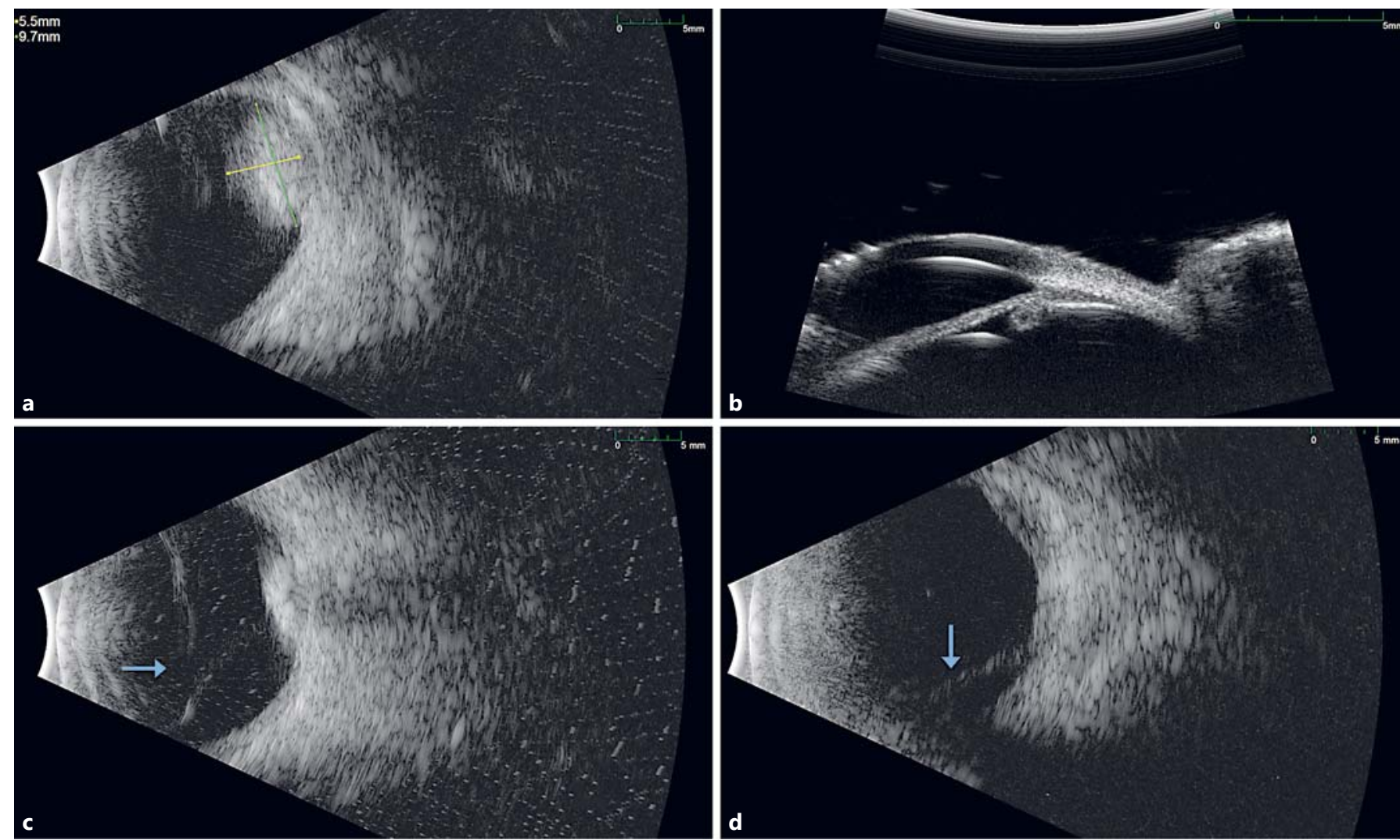

Fig. 3. a B-scan at diagnosis for patient 2 showing a dome-shaped retinal tumor with intralesional calcification consistent with retinoblastoma which measures $5.5 \times 9.7 \mathrm{~mm}$. The hyaloid appears to be attached over the tumor. b Ultrasound biomicroscopy image done before intravitreal injection in patient 2 showing no anterior

involvement by the tumor or seeding. c B-scan after the retinal toxicity was noted in patient 2 showing detachment of the posterior hyaloid (arrow). d Similar B-scan done after toxicity was noted in patient 1 showing detachment of the posterior hyaloid (arrow).
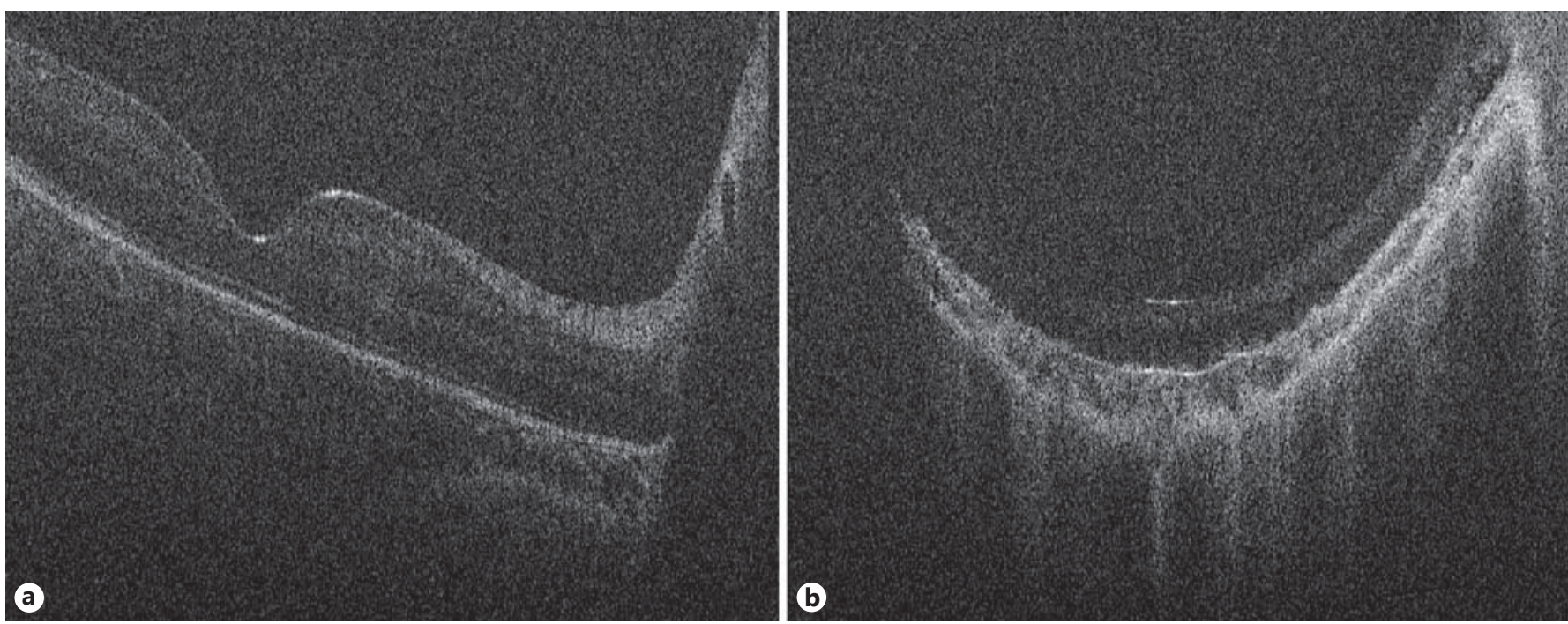

Fig. 4. a OCT performed before injection on patient 2 showing a normal foveal contour without direct involvement of the macula by the tumor or seeding. b Subsequent OCT performed 2 months

after the toxicity had been noted showing diffuse inner and outer retinal atrophy, loss of the foveal depression, and atrophy of the underlying choroid. 


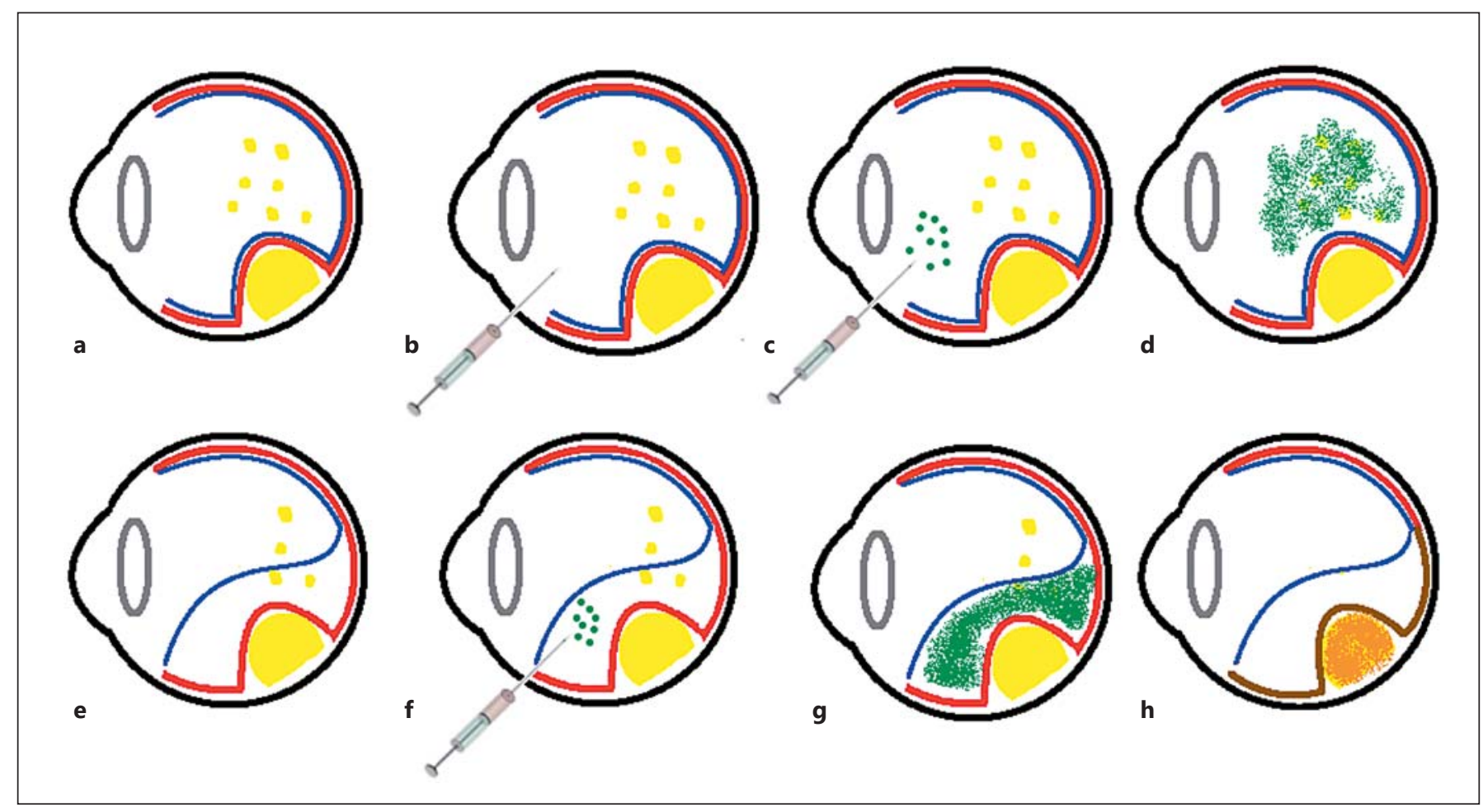

Fig. 5. a Schematic rendition of an eye with retinoblastoma and associated vitreous seeding. The blue line represents the hyaloid face, the red line represents the retina, and the yellow mass represents the tumor. b-d Injection of melphalan (green) into the vitreous cavity via the pars plana. e Localized posterior hyaloid detach- ment over the tumor. $\mathbf{f}, \mathbf{g}$ Injection of melphalan into the subhyaloid space due to the presence of partial detachment. $\mathbf{h}$ Treated retinoblastoma (orange) with retinal atrophy (brown line) and resolution of vitreous seeding.

After a critical evaluation of the present cases, no clear, single etiological factor for the chorioretinal toxicity could be identified. An initial consideration was that the toxicity might have been related to inadvertent injection of a higher melphalan dose secondary to a pharmaceutical compounding error. But in the case of patient 1 , the other eye served as an internal control as it was injected on the same day, with a higher dose, and suffered no complication. Another plausible explanation is that the toxicity was due to a technique-related mechanism. In the present cases, 2 separate ophthalmologists, both following the technique described in the original papers by $\mathrm{Mu}-$ nier et al. [5, 9], injected 1 of the 2 patients. In both cases, this was the second intravitreal melphalan injection, and no apparent intraoperative or immediate postoperative complications were noted. Given that 2 different physicians performed the injections, this makes the complication less likely to be directly related to the technique applied, even if it may still have played a role. Patient-specific characteristics also may have contributed to the 
toxicity. Both patients were less than 12 months of age at the time of the injection. However, the first injections were well tolerated, and the contralateral eye of patient 1 did not show any signs of toxicity. Additionally, at our center, 9 other eyes of patients less than 12 months of age were injected without any signs of toxicity.

In our opinion, the leading hypothesis regarding the cause of the acute toxicity is an anatomic change in the vitreoretinal interface and the absence of an attached posterior hyaloid to protect the retina. Previous reports have shown that routine intravitreal injections in adults can induce posterior vitreous detachment in up to $25 \%$ of patients [10]. Moreover, endophytic tumors with vitreous seeding cause apical disruption of the internal limiting membrane/hyaloid complex, contributing to the change of the vitreoretinal interface surrounding the tumor [4]. Thus, we hypothesize that in these cases melphalan was injected into the retrohyaloid space, causing the neurosensory retina to be directly exposed to a higher concentration of the drug, leading to acute retinopathy (fig. 5). Two clinical aspects seen in our cases support this theory. First, there is a sharp demarcation between the area of retinal atrophy and the normal retina, suggesting a localized, physical mechanism for the toxicity, such as the drug being injected into the retrohyaloid space. Secondly, both patients were noted to have posterior vitreous detachment on B-scan ultrasonography after the toxicity had been noted (fig. 3). Moreover, a recent case report described similar hemorrhagic toxicity from an intravitreal injection given as close to the retina as possible to increase the concentration of melphalan at the retinal surface in order to treat subretinal disease [11]. Despite this compelling evidence, an alternate potential mechanism increasing the concentration of melphalan in contact with the posterior retina could result from injecting the drug directly into Erggelet's retrolental space or into Cloquet's canal. However, in such a case, the retinal toxicity would be confined to Martegiani's area around the optic nerve head, with possible extension to the bursa premacularis.

The clinical presentation of retinal edema with diffuse hemorrhages, vascular occlusion, sheathing, and capillary nonperfusion, similar to the cases herein presented, has been previously described with acute aminoglycoside toxicity [12]. One proposed mechanism for aminoglycoside toxicity is a vasoocclusive event, which has also been noted to occur more often after repetitive injection in non-vitrectomized eyes [12]. This may suggest a mechanism of toxicity similar to that hypothesized here, which leads to a secondary vasoocclusive event. Further sup-

Acute Hemorrhagic Retinopathy after

Melphalan Injection for Retinoblastoma porting this hypothesis is the loss of foveal depression and diffuse inner retinal atrophy seen on post-toxicity OCT (fig. 4), which is similar to that seen late after central retinal artery occlusion. However, other studies suggest that aminoglycoside toxicity is not fully explained by a vascular mechanism and there may be a mixed-mechanism etiology for this toxicity as well [13].

At the CHLA, we have performed 137 intravitreal injections in 36 eyes since 2013, and 2 eyes have developed this serious retinal toxicity. While we believe that intravitreal melphalan injection is a relatively safe and effective way of treating vitreous seeds in advanced retinoblastoma, ocular oncologists using this treatment must be aware of this potential toxicity. We hypothesize that this complication results from disruption of the vitreoretinal interface, likely induced by an endophytic tumor and/or the initial intravitreal injection. Therefore, we recommend that B-scan ultrasonography be performed prior to each intravitreal melphalan injection to evaluate whether posterior vitreous detachment occurs. If present, we recommend that the treating physician take additional care during injection to follow the protocol as described by $\mathrm{Mu}$ nier et al. [5], modified as follows. We recommend that injection be done with a $12-\mathrm{mm}(0.5$-inch), 32- or 33gauge needle. This is to ensure perforation of the hyaloid and intravitreous injection which will prevent the drug from concentrating in the retrohyaloid space. During injection the needle should remain in the mid-to-anterior vitreous and be visible about $1 \mathrm{~mm}$ behind the intraocular lens. Furthermore, during injection the surgeon should visualize a cyst or bubble formation which confirms intravitreal drug delivery. Finally, an increased concentration may contribute to retinal toxicity. Although this is unproven, we recommend injections with a concentration of melphalan of $20 \mu \mathrm{g} / 0.1 \mathrm{ml}$.

As this effective treatment modality continues to be used with greater frequency, we will gain a better understanding of its best indications and toxicities. We strongly believe that reporting the toxicity seen in these 2 patients is essential to better understand the treatments used for retinoblastoma as well as to appropriately counsel families regarding potential risks of intravitreal melphalan injections.

\section{Statement of Ethics}

This research complies with the guidelines on human studies and animal welfare regulations. The subjects herein described gave their informed consent, and the study protocol was approved by the Committee on Human Research at the CHLA. 


\section{Disclosure Statement}

The authors do not have any conflicts of interest to disclose.

\section{References}

1 Ericson LA, Rosengren BH: Present therapeutic resources in retinoblastoma. Acta Ophthalmol (Copenh) 1961;39:569-576.

2 Kaneko A, Suzuki S: Eye-preservation treatment of retinoblastoma with vitreous seeding. Jpn J Clin Oncol 2003;33:601-607.

3 Francis JH, Schaiquevich P, Buitrago E, et al: Local and systemic toxicity of intravitreal melphalan for vitreous seeding in retinoblastoma: a preclinical and clinical study. Ophthalmology 2014;121:1810-1817.

4 Munier FL: Classification and management of seeds in retinoblastoma. Ellsworth Lecture Ghent August 24th 2013. Ophthalmic Genet 2014;35:193-207.

5 Munier FL, Gaillard MC, Balmer A, et al: Intravitreal chemotherapy for vitreous disease in retinoblastoma revisited: from prohibition to conditional indications. $\mathrm{Br} \mathrm{J}$ Ophthalmol 2012;96:1078-1083.
6 Ghassemi F, Amoli FA: Pathological findings in enucleated eyes after intravitreal melphalan injection. Int Ophthalmol 2014;34:533540

7 Smith SJ, Smith BD, Mohney BG: Ocular side effects following intravitreal injection therapy for retinoblastoma: a systematic review. Br J Ophthalmol 2014;98:292-297.

8 Suzuki S, Aihara Y, Fujiwara M, Sano S, Kaneko A: Intravitreal injection of melphalan for intraocular retinoblastoma. Jpn J Ophthalmol 2015;59:164-172.

9 Munier FL, Soliman S, Moulin AP, Gaillard MC, Balmer A, Beck-Popovic M: Profiling safety of intravitreal injections for retinoblastoma using an anti-reflux procedure and sterilisation of the needle track. Br J Ophthalmol 2012;96:1084-1087.
10 Geck U, Pustolla N, Baraki H, Atili A, Feltgen $\mathrm{N}$, Hoerauf H: Posterior vitreous detachment following intravitreal drug injection. Graefes Arch Clin Exp Ophthalmol 2013;251:16911695.

11 Francis JH, Marr BP, Brodie SE, Gobin P, Dunkel IJ, Abramson DH: Intravitreal melphalan as salvage therapy for refractory retinal and subretinal retinoblastoma. Retin Cases Brief Rep 2015, Epub ahead of print.

12 Penha FM, Rodrigues EB, Maia M, et al: Retinal and ocular toxicity in ocular application of drugs and chemicals - part II: retinal toxicity of current and new drugs. Ophthalmic Res 2010;44:205-224.

13 Hancock HA, Guidry C, Read RW, Ready EL, Kraft TW: Acute aminoglycoside retinal toxicity in vivo and in vitro. Invest Ophthalmol Vis Sci 2005;46:4804-4808. 